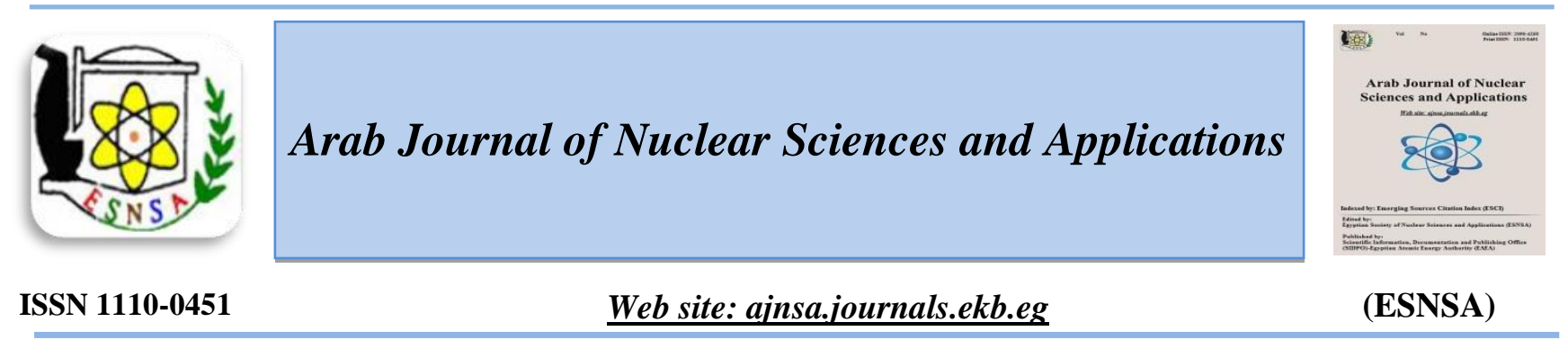

\title{
The Effect of the Interface on Magnetic Properties of Perovskite-Spinel Nanocomposites
}

\author{
E. H. El-Khawas \\ Basic Science Department, Higher Technological Institute, Tenth of Ramadan City, Egypt
}

\begin{abstract}
Received 31 ${ }^{\text {st }} \mathrm{Jan} 2019$ In this research, perovskite-spinel nanocomposites (of the synthsiesed formula (x) $\left(\mathrm{Cu}_{0.3} \mathrm{Mn}_{0.7} \mathrm{Fe}_{2} \mathrm{O}_{4}\right)-(1-$ Accepted $\left.26^{\text {th }} \mathrm{Jun} 2019 \mathrm{x}\right)\left(\mathrm{La}_{0.90} \mathrm{Bi}_{0.10} \mathrm{FeO}_{3}\right) ; 0 \leq \mathrm{x} \leq 1$, were prepared using the auto-combustion method. The fundamental phases of orthorhombic for (La0.90Bi0.10FeO3(LBFO) and the cubic phases for Cuo.3Mn0.7Fe2O4(CMFO) were demonstrated utilizing X-Ray Diffractometry (XRD) analysis.. Highresolution transmission electron microscopy (HRTEM) images reveal nearly spherical nanoparticles for the parents compositions and agglomeratation for nanocomposites. Magnetic hysteresis loops demonstrate ferromagnetic (FM) behavior for the spinel compound and nanocomposite, where LBFO possesses G-type antiferromagnetic (AFM). Competition between FM and AFM phases leads to enhancement in magnetic parameters such as saturation magnetization (Ms), remnant magnetization (Mr) and coercivity (Hc). The magnetic properties at low temperatures show a decrease in the magnetization and coercivity with increasing the temperature while the magnetic exchange bias $\mathrm{H}_{\mathrm{EB}}$ increased until $\mathrm{T}=\mathbf{2 0 0} \mathrm{K}$ and then, decreased.

The intergranular magnetostatics interactions have promoted along the LBFO and CMFO nanocomposites interfaces which play a decisive role in strain energy, exchange energy, and magnetic anisotropy.
\end{abstract}

Keywords: nanocomposites; perovskite-spinel; magnetic properties; exchange interaction; exchange bias

\section{Introduction}

Assembly of different substances is one of scientific approaches for material science development to obtain new desirable physical characterizations. This can be utilized in several industrial applications that use modified materials with advanced specifications. Nanocomposites of metal oxide are developed to create novel materials with high performance as well as improvement of their properties and behavior [1-8]. Composite interfaces play the main role in the improving functionality of materials and modulating its effective properties [9]. The properties of new composites result from the interaction of their component phases along the grains boundaries interfaces which enhance the key role of interfacial reaction [10]. Several phenomena occur at the interface has been reported in previous researches, i.e. rearrangement of chemical bonding [11]; spincharge and orbital reconstruction [12]; modifications to the electronic structure [13]. The incorporation of spinel and perovskite seems to be very promising for making spinel- perovskite systems with multiferroic or multifunctional properties [14].

One of the great interests also is the perovskitespinel interface in complex oxide based magnetic tunnel junctions [10]. A perovskite structure of $\mathrm{LaFeO}_{3}$ (LFO) nanocomposites was marked by significant properties, hence, it was employed in

Corresponding author: enash12@hotmail.com

DOI: 10.21608 /ajnsa.2019.7827.1176

(C) Scientific Information, Documentation and Publishing Office (SIDPO)-EAEA 
applications of superlattices $[15,16]$. Additionally, the combination of perovskite structure with a spinel system has produced an amazing property and revealing anomalous phenomena..

It is also known that the LFO is a member of the rare earth orthoferrite family. It has an orthorhombic perovskite structure (space group: Pnma). This exhibits a phase transition from orthorhombic to rhombohedral at $\mathrm{T}=1260 \mathrm{~K}$ [17]. In addition, LFO possesses G-type antiferromagnetism ordering arises as a result of indirect exchange interaction between $\mathrm{Fe}^{3+}$ ions through $\mathrm{O}^{2-}$ ions $[18,19]$. The $\mathrm{R} 3 \mathrm{c}$ in rhombohedral symmetry allows the presence of the weak ferromagnetic moment due to spin canting configuration [20-24]. Lanthanum orthoferrite $\left(\mathrm{LaFeO}_{3}\right)$ is recognized high value of Néel temperature $\left(\mathrm{T}_{\mathrm{N}} \sim 1013 \mathrm{~K}\right)$ as compared with the antiferromagnetic material $[25,26]$. Otherwise, Spinel ferrite, $\mathrm{MFe}_{2} \mathrm{O}_{4}\left(\mathrm{M}=\mathrm{Cu}^{2+}, \mathrm{Co}^{2+}, \mathrm{Ni}^{2+}, \mathrm{Fe}^{2+}\right.$, $\mathrm{Zn}^{2+}$ etc) nanocrystals has attracted much attention due to their distinctive properties [27-30]. Some of the main distinguishing features of the spinel ferrites are the high permeability, high electrical resistivity, and low eddy current at high-frequency electromagnetic wave transmission. These properties make them convenient for different technological applications such as telecommunication and microwave devices, magnetic fluids, high-density magnetic storage, drug delivery, and gas sensors. $\mathrm{CuFe}_{2} \mathrm{O}_{4}$ is one of the famous soft ferrimagnetic insulators with an inverse spinel structure, which exists in tetragonal and cubic structures. Tetragonal structures of those compounds are stabilized at room temperature. They transform into cubic structure at a temperature within $633 \mathrm{~K}$ or higher due to JahnTeller distortion. The distortion affected the magnetic properties, where the cubic structure is characterized by the presence of a large magnetic moment than that of the tetragonal one. This is because cubic structure has more cupric ions $\left(\mathrm{Cu}^{2+}\right)$ at tetrahedral sites as compared to that in the case of tetragonal structure $[31,32]$. In a previous work by the author, the two systems $\mathrm{La}_{1}$. ${ }_{x} \mathrm{Bi}_{x} \mathrm{FeO}_{3}$ (LBFO) and $\mathrm{Mn}_{1-\mathrm{x}} \mathrm{Cu}_{\mathrm{x}} \mathrm{Fe}_{2} \mathrm{O}_{4}$ (CMFO) were synthesized by auto combustion method[33]. The magnetic properties demonstrated that the concentration $\mathrm{La}_{0.9} \mathrm{Bi}_{0.1} \mathrm{FeO}_{3}$ and $\mathrm{Mn}_{0.7} \mathrm{Cu}_{0.3} \mathrm{Fe}_{2} \mathrm{O}_{4}$ have the maximum value of magnetization. In this work, perovskite-spinel nanocomposites were synthesized for enhancement the multiferroicity property for CMFO and LBFO materials. On the other hand, the perovskite-spinel interface effect was investigated.

\section{Experimental Work \\ Preparation of Samples}

The parent compounds of the nanocomposites namely, $\mathrm{La}_{0.90} \mathrm{Bi}_{0.10} \mathrm{Fe}_{2} \mathrm{O}_{3} \quad$ (LBFO) and $\mathrm{Cu}_{0.3} \mathrm{Mn}_{0.7} \mathrm{Fe}_{2} \mathrm{O}_{4}$ (CMFO) were prepared using the sol-gel auto-combustion method as reported earlier [33-35]. The nanocomposites (NCs) were prepared from the $\mathrm{LBFO}$ and CMFO in four different weight ratios according to the formula: (x)CMFO(1-x)LBFO, where $\mathrm{x}=0,0.2,0.4,0.6,0.8$ and 1 . To prepare the nanocomposites, the assigned weight per gram of the two components were mixed together and ground for $3 \mathrm{~h}$ to allow good mechanical mixing and finally annealed at $400^{\circ} \mathrm{C}$ for further $2 \mathrm{~h}$ with a heating/cooling rate of 5 ${ }^{\circ} \mathrm{C} / \mathrm{min}$.

\section{Characterization}

Structural characterization of NCs was carried out using a Bruker D8 Advance X-ray diffractometer (XRD) with $\mathrm{Cu} \mathrm{K}_{\alpha}$ radiation $(\lambda=1.5418 \AA$ ). The microstructure and morphology of the nanoparticles were studied using a JEOL-2100 high-resolution transmission electron microscope (HRTEM). Magnetic measurements were carried out at room temperature using a vibrating sample magnetometer (VSM; Lake Shore -7410-USA) with a maximum magnetic field of $20 \mathrm{kG}$.

\section{Results and Discussion}

\section{Microstructure analysis}

Figure (1) shows the XRD patterns of (x) CMFO (1-x) LBFO ( $x=0.0,0.2,0.4,0.6,0.8$ and 1$)$ at room temperature. It could be seen that the composite formula to incorporate the spinel ferrite and the orthoferrite structures has greatly affected each other. At $\mathrm{x}=0$, the spinel structure is absent ,but all orthoferrite peaks of LBFO composition have been displayed in the XRD pattern. All peaks for $\mathrm{x}=0$ in $\mathrm{XRD}$, show a pure phase orthorhombic structure with space group (Pbnm) as indexed with ICCD card No.74-2203. On the other hand, at $\mathrm{x}=1$, the orthoferrite disappeared completely. Therefore, formation of the single phase for crystalline spinel (CMFO) has confirmed the formation of cubic structure (ICDD card No. 770010) in which it confirmed_the cubic structure. 
All diffracted peaks of the nanocomposites shown in Figure(1) are identified as due to different crystal planes of orthorhombic structure LBFO (JCPDS card no. 74-2203) and cubic spinel CMFO (JCPDS card no. 77-0010) phases. As predictable, with increasing CMFO weight ratio, the diffraction peaks of CMFO increased in strength gradually. Hence, it was observed that the diffraction peaks intensities of the two parents agree well with the change of their respective ratios in the nanocomposites.

The crystallite size of the two parents LFBO and CMFO were evaluated according to the_broadening $\mathrm{X}$-ray line of the maximum intensity peak utilizing the Scherrer's formula: $D=0.89 \lambda / \beta \cos \theta[36,37]$. Where $\lambda$ is the $X$-ray wavelength $\left(0.15406 \mathrm{~nm}\right.$ for $\left.\mathrm{Cuk}_{\alpha}\right) ; \theta$ is the Bragg's, D is the crystallite size and $\beta$ is the full width broadening at half maximum (FWHM) in radians of the diffraction peak. The crystallite size was 23 and $15 \mathrm{~nm}$ for LBFO and CMFO, respectively. However, the small shift occurring in the peaks of some samples does not indicate that the main structures of synthesized nanocomposites have changed. This behavior might occur as a result of increasing the spinel concentration on the expense of concentration of the orthoferrite structure.

The morphological features and particle sizes distribution of compositions ( $\mathrm{x}=0.0,1.0,0.4$ and 0.8 ) have been examined using HR-TEM measurements, as shown in Figure 2. The two plain compositions have displayed nearly spherical nanoparticles with an average particle sizes of 28 $\mathrm{nm}$ for LBFO and $17 \mathrm{~nm}$ for CMFO, these values have agreed with those calculated from XRD analysis.. Moreover, the electronic diffraction subimages have shown a good crystallinity nature for the two parent's compositions.

The HR-TEM images for compositions $\mathrm{x}=0.4$, and 0.8 have revealed an agglomerated nanoparticle and polygons geometric shapes. This observation may be due to the physical interaction along the interfacial boundaries between ferromagnetism nanoparticle and antiferromagnetism nanoparticles.

\section{Magnetic properties}

Figure 3 depicts the magnetic hysteresis loops curves of $\mathrm{CMFO}_{(\mathrm{x})}-\mathrm{LBFO}_{(1-\mathrm{x})}(\mathrm{x}=0.0,0.2,0.4,0.6$, 0.8 and 1) utilizing a vibration sample magnetometer (VSM) instrument with applying magnetic field intensity $\pm 20 \mathrm{kG}$ at room temperature.

Furthermore, Table 1 records the results of magnetic parameters of prepared nanocomposites such as coercivity $\mathrm{Hc}$, remnant magnetization $\mathrm{Mr}$, saturation magnetization Ms, magnetic anisotropy energy, and squareness ratio $\mathrm{R}$.

The inset graphs in Figure 3 focus on the behavior curve of $\mathrm{M}-\mathrm{H}$ with regard to small values of the magnetic field. Obviously, the M-H curve of LBFO ( $x=0)$ have exhibited a weak ferromagnetic property with very small remnant magnetization $\operatorname{Mr}(0.265 \mathrm{emu} / \mathrm{g})$.

In fact, pure LBFO has the kind of G-type AFM with canted $\mathrm{Fe}^{3+}$ spins [38-40]. The weak ferromagnetism is due to the fractional alignment of the canted $\mathrm{Fe}^{3+}$ spins. Moreover, magnetic hysteresis curve of CMFO shows a high saturation magnetization Ms (37.96 emu/g). Therefore, the nature of the magnetic ordering plays a main role in ferrimagnetic behavior for CMFO as expected. Non zero values of squareness ratio $R$ for (x)CMFO-(1-x)LBFO NCs have demonstrated the existence of intergranular magnetostatic interactions between the LBFO and CMFO along the interfacial grains' boundaries. In addition, based on Vegard's law approximation, the quantity of

of intergranular magnetostatics interactions of the prepared nanocomposites could calculated by equation (1)[41, 42].,

$\mathrm{M}_{\mathrm{cal}}=(1-\mathrm{x}) \mathrm{M}_{\mathrm{LBFO}}+(\mathrm{x}) \mathrm{M}_{\mathrm{CMFO}}$

Where $M_{\text {cal }}$ is calculated values of $M r, M_{L B F O}$ and $\mathrm{M}_{\text {CMFO }}$ are the measured values of $\mathrm{Mr}$ of the LBFO and CMFO pure phases, respectively. The measured and calculated values of $\mathrm{Mr}$ are listed in Table1. It is noted that the measured values of $\mathrm{Mr}$ for nanocomposites are lower than the calculated ones excepted at $(x=0.4)$. This indicates that the spin alignment at the interfacial grain boundaries interfaces for the CMFO (x) - LBFO (1-x) nanocomposites have been modified as compared to their individual pure phases. The magnetically coupled interactions at the interface of nanocomposites play a decisive role in strain energy, exchange energy, and magnetic anisotropy. The origin of these magnetic interactions near the interface of two various magnetic substances, when they are nearby to each other, has been theoretically well investigated in the literature [4346]. The saturation magnetization Ms of the NCs increases linearly with increasing (CMFO) content as shown in Figure(4). This is attributed to the high magnetic moment of CMFO phase. The coercivity $\left(\mathrm{H}_{\mathrm{c}}\right)$ increases with CMFO content reaching a maximum value at $\mathrm{x}=0.4$ and then, decreases. 
Additionally, the anomalous behavior could be observed at the composition $(\mathrm{x}=0.4)$, in which $\mathrm{Hc}$ value is higher than the values of other concentrations. Furthermore, the exchange bias differs completely from the above and below concentrations which urge the author to conduct further studies.

Hence, the magnetic exchange interaction occurs at this concentration between ferromagnetic and antiferromagnetic. Otherwise, the anisotropic magnetic energy (area erg/g) and squareness ratio (R) have augmented with increasing (CMFO) content as shown in Table (1). This results in reorientation tilting, that gave a rise to the distortion, which is due to the existence of two different magnetic orders (antiferromagnetism and ferrimagnetism) [47].

Figure (5) shows the variation of the magnetic hysteresis loop of NCs sample with $\mathrm{x}=0.4$ at different absolute temperatures $(100,150,200$, $250,300 \mathrm{~K})$. The inset graph of the middle region of the hysteresis loop curves vary with the testing temperature variations. This behavior assures the exchange bias in the midpoint of the magnetic field axis.

The data of $\mathrm{M}_{\mathrm{s}}, \mathrm{M}_{\mathrm{r}}, \mathrm{H}_{\mathrm{c}}$ are summarized in Table (2). The drastically reduced in the saturation magnetization $\mathrm{Ms}$ and coercivity $\mathrm{H}_{\mathrm{c}}$ with increasing temperature are shown in Figure (6). It is worth to mention that whenever the temperature increases, more thermal energy is provided and the spins of individual electrons that gain a higher energy states resulting in random directions and less alignment with their neighbors. This phenomenon leads to reducing the total magnetization. Furthermore, a small field is required to reduce the remnant magnetization to be zero value which leads to a decrease in the coercivity $\mathrm{H}_{\mathrm{C}}$ values $[48,49]$. [48,49].

The phenomenon of exchange bias (EB) is associated with the magnetic exchange anisotropy to control the relation between ferromagnetic and antiferromagnetic FM/AFM substances at the interface of them, indicating a shift of hysteresis loop along the magnetic field axis [50,51]. The existence of EB in any material is due to the coexistence of magnetic coupling between antiferromagnetic and ferromagnetic orders [52, 53]. A different testing temperatures, the values of the exchange bias field $\left(\mathrm{H}_{\mathrm{EB}}\right)$ are calculated from the horizontal displacement in the midpoint at the hysteresis loop of the specific concentration $(x=0.4)$. Table 2 shows the change in values of $\mathrm{H}_{\mathrm{EB}}$ at different temperatures, where the value of $\mathrm{H}_{\mathrm{EB}}$ rapidly increases from $100 \mathrm{~K}$ up to $200 \mathrm{~K}$ and then quickly decreases again from 200 up to 300 $\mathrm{K}$.. One possible explication is the EB phenomenon is emerging from the synthesized nanometric systems, where the uncompensated surface spins favor the AFM coupling and the core of particle favor FM coupling. This causes a creation of natural AFM/FM interfacial boundaries interfaces and leads to EB effect [54,55]. Exchange bias commonly takes place in multilayers or bilayers of magnetic nanostructures when an antiferromagnetic material with hard magnetization characteristic causes a shift in the soft magnetization hysteresis loop curve of a ferromagnetic material [56-58].

This implies that the sample of $x=0.4$ may be considered a multilayers magnetic system which has internal magnetic exchange interaction between interfacial boundaries interfaces of AFM/FM. Lastly, the EB plays an important role and effectiveness in many electronic applications such as ultrahigh-density magnetic recording, giant magnetoresistance, and spin valve devices [59-61].

\section{Conclusion}

The perovskite and spinel nanocomposites were successfully prepared using the sol-gel autocombustion method. XRD patterns confirmed a single phase for the parents LBFO and CMFO. The nanocomposite perovskite-spinel phase has XRD peaks that vary in intensity depending on the variation of their respective weight ratios in the nanocomposites. HRTEM images demonstrated nanostructure for $\mathrm{LBFO}$ and CMFO with a particle size of 28 for LBFO and $17 \mathrm{~nm}$ respectively. The electron diffraction showed a good crystallinity for the two parents and NCs. The perovskite-spinel interfaces has impacted the magnetic behavior of the (x)CMFO-(1-x) LBFO bulk composites and this behavior has been confirmed by $\mathrm{M}-\mathrm{H}$ curves.. An enhancement in the magnetization, coercivity and squareness ratio was observed with CMFO content in composite systems. The magnetic properties show a decrease in magnetization and coercivity with increasing the temperature while the magnetic exchange bias $\mathrm{H}_{\mathrm{EB}}$ was increased until $\mathrm{T}=200 \mathrm{~K}$ and then, decreased. 
Table (1): The values of saturation magnetization $(M s)$, remnant magnetization $\left(M_{r}\right)$, coercicvty $\left(H_{c}\right)$, exchange bias field $\left(H_{\mathrm{EB}}\right)$, squareness ratio $(\mathrm{R})$ and calculated remnant magnetization $\left(\mathrm{Mr}_{\mathrm{Cal}}\right)$ at room temperature for the nanocomposition (x)CMFO-(1-x)LBFO

\begin{tabular}{cccccccc}
\hline Conc. $x$ & $\begin{array}{c}\mathrm{M}_{\mathrm{s}} \\
\mathrm{emu} / \mathrm{g}\end{array}$ & $\begin{array}{c}\mathrm{M}_{\mathrm{r}} \\
\mathrm{emu} / \mathrm{g}\end{array}$ & $\begin{array}{c}\mathrm{H}_{\mathrm{c}} \\
(\mathrm{G})\end{array}$ & $\begin{array}{c}\mathrm{H}_{\mathrm{EB}} \\
(\mathrm{G})\end{array}$ & $\mathrm{R}$ & $\begin{array}{c}\text { Area } \\
\mathrm{erg} / \mathrm{g}\end{array}$ & $\begin{array}{c}\mathrm{Mr}_{\mathrm{Cal}} \\
\mathrm{emu} / \mathrm{g}\end{array}$ \\
\hline 0 & 3.37 & 0.27 & 68 & -14.6 & 0.08 & 698 & ----- \\
0.2 & 8.41 & 1.83 & 125 & -3.45 & 0.22 & 3175 & 2.23 \\
0.4 & 17.39 & 4.38 & 149 & 3.76 & 0.25 & 9177 & 4.19 \\
0.6 & 23.82 & 5.92 & 134 & -2.38 & 0.25 & 11020 & 6.15 \\
0.8 & 31.5 & 7.96 & 140 & 3.43 & 0.26 & 15610 & 8.11 \\
1 & 37.96 & 10.07 & 145 & -3.94 & 0.27 & 18900 & ---- \\
\hline
\end{tabular}

Table (2): The magnetic parameters for the nanocomposition (x)CMFO-(1-x)LBFO. for $\mathrm{x}=0.4$ at different temperature

\begin{tabular}{ccccc}
\hline $\mathrm{T}(\mathrm{K})$ & $\mathrm{Ms}(\mathrm{emu} / \mathrm{g})$ & $\mathrm{Mr}(\mathrm{emu} / \mathrm{g})$ & $\mathrm{Hc}(\mathrm{G})$ & $\mathrm{HEB}(\mathrm{G})$ \\
\hline 100 & 21.162 & 6.81 & 415 & 4.3 \\
150 & 20.371 & 5.52 & 344 & 5.21 \\
200 & 19.304 & 4.34 & 295 & 22.4 \\
250 & 18.290 & 3.77 & 255 & 16.28 \\
300 & 17.272 & 2.92 & 218 & 4.76 \\
\hline
\end{tabular}

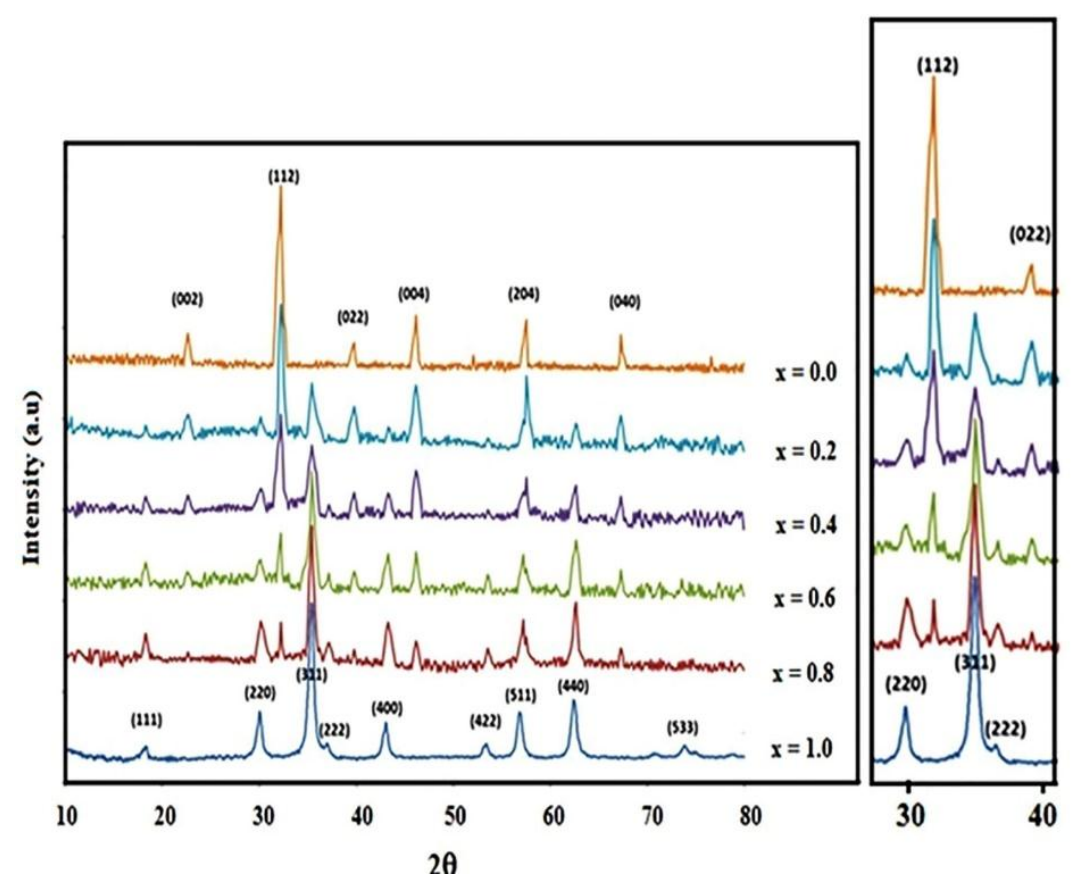

Figure (1): X-ray diffraction (XRD) patterns of (x)CMFO-(1-x)LBFO $(x=0.0,0.2,0.4,0.6$ and 1.0) at room temperature 


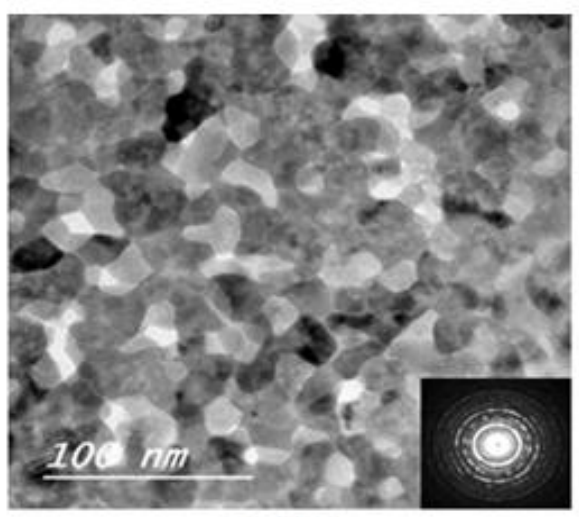

$\mathbf{x}=\mathbf{0 . 0}$

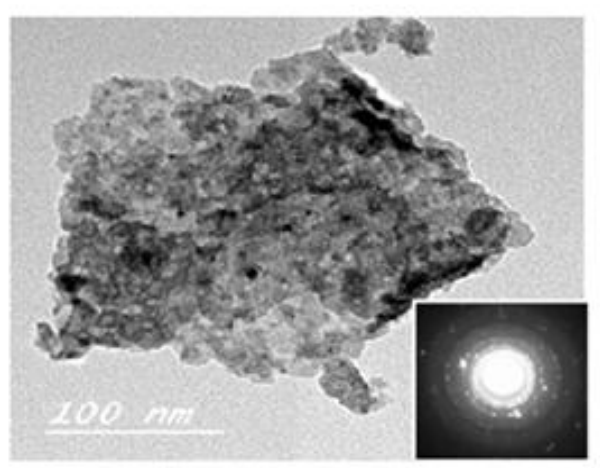

$x=0.4$

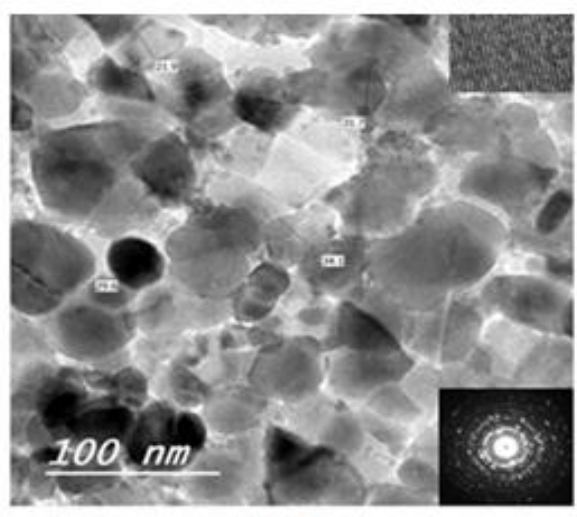

$\mathbf{x}=\mathbf{1 . 0}$

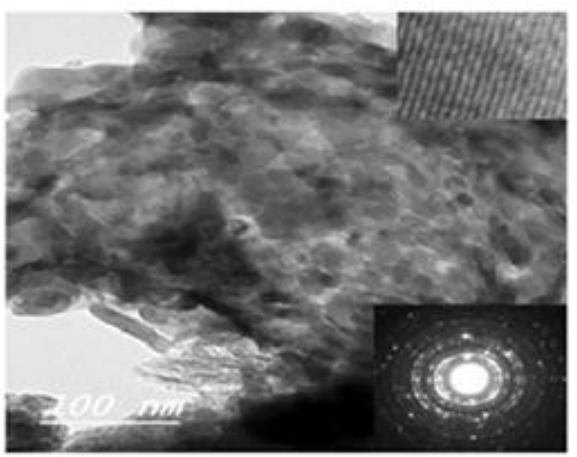

$x=0.8$

Figure (2): HRTEM images and electron diffraction patterns of nanocomposite (x)CMFO-(1-x)LBFO.

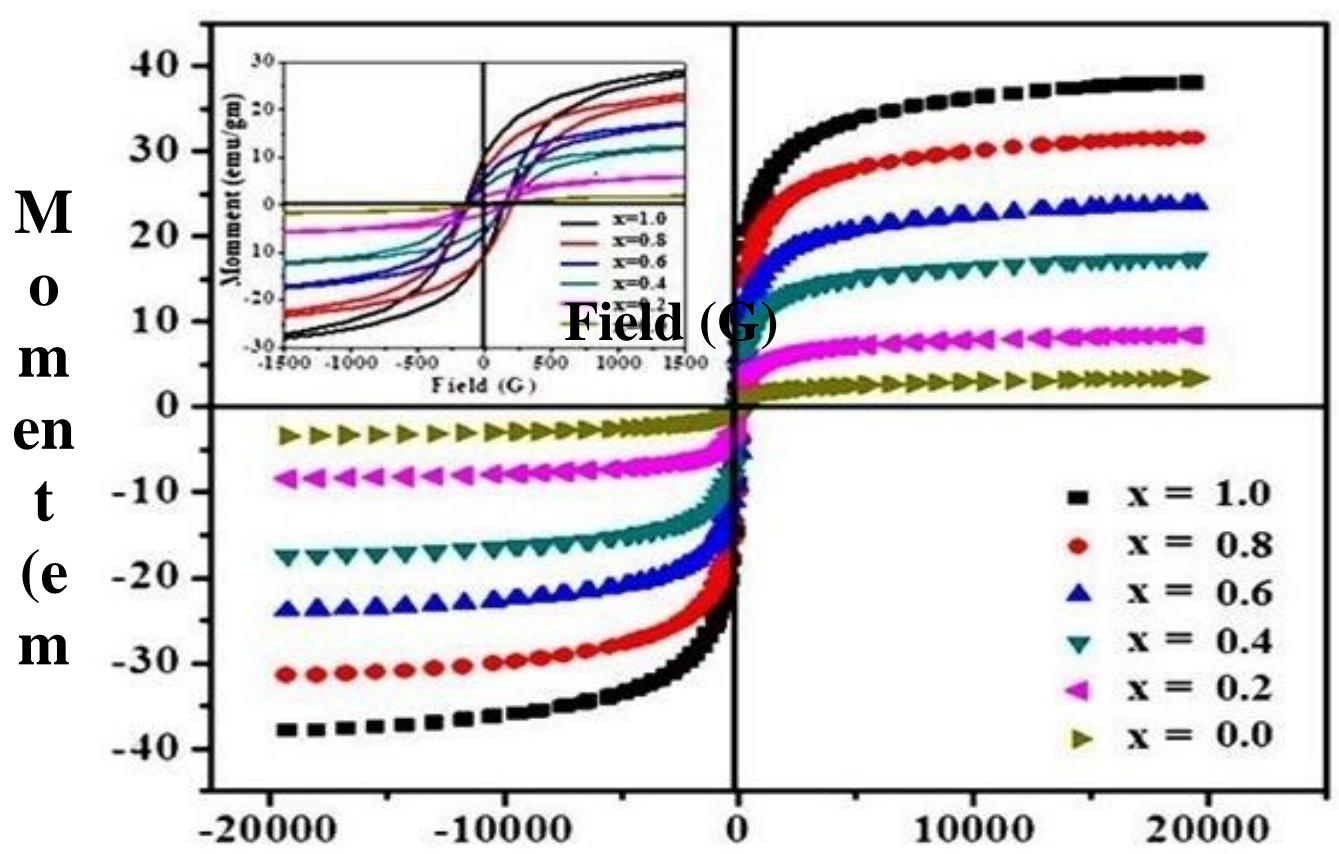

Figure (3): Magnetic hysteresis loops of (x)CMFO-(1-x)LBFO ( $x=0.0,0.2,0.4,0.6,0.8$ and 1.0) at room temperature. Inset: The middle region of the (VSM) of all concentrations 


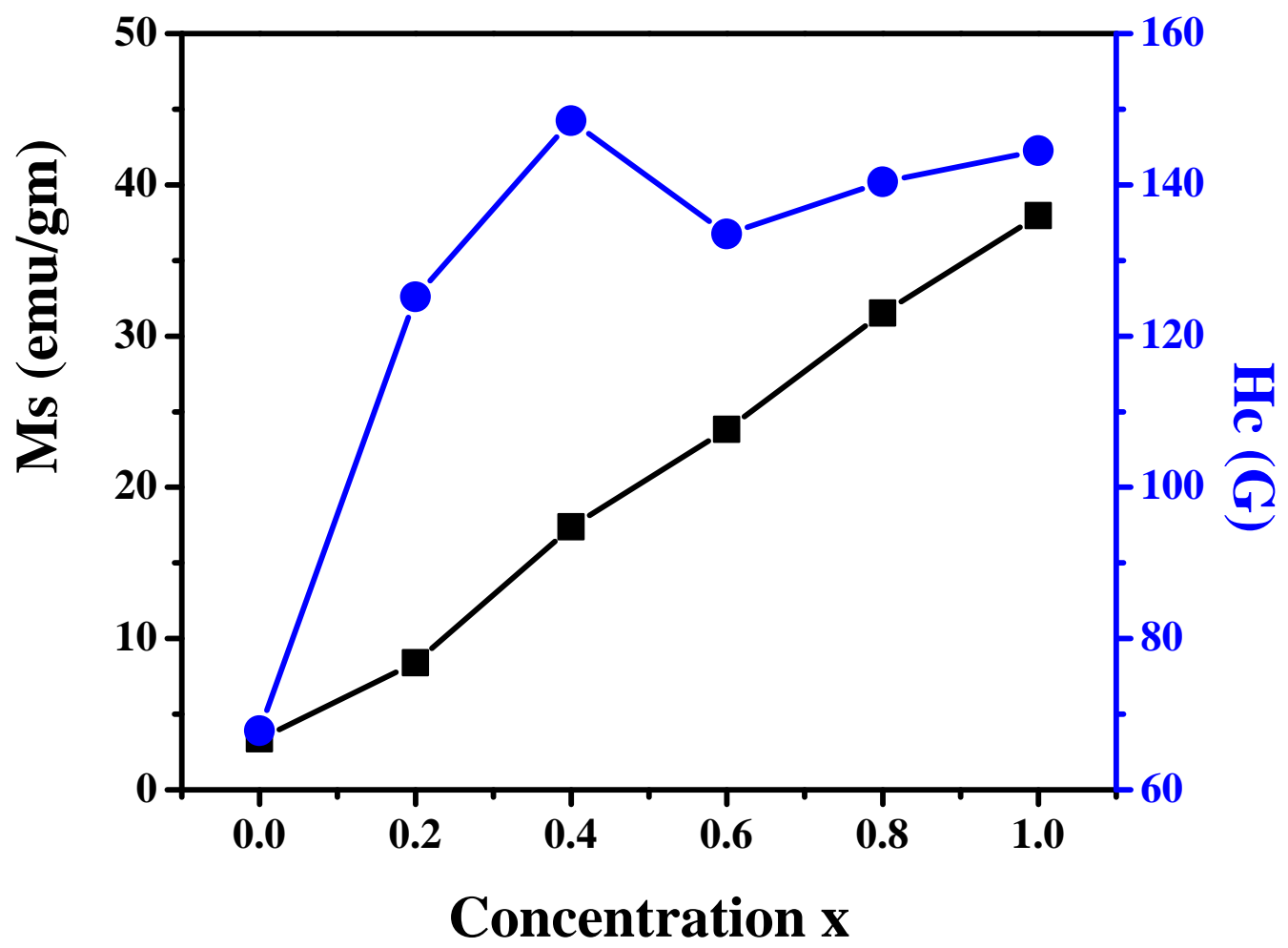

Figure (4): Variation of saturation magnetization (Ms) and coercivity (Hc) of (x)CMFO-(1-x)LBFO with CMFO content

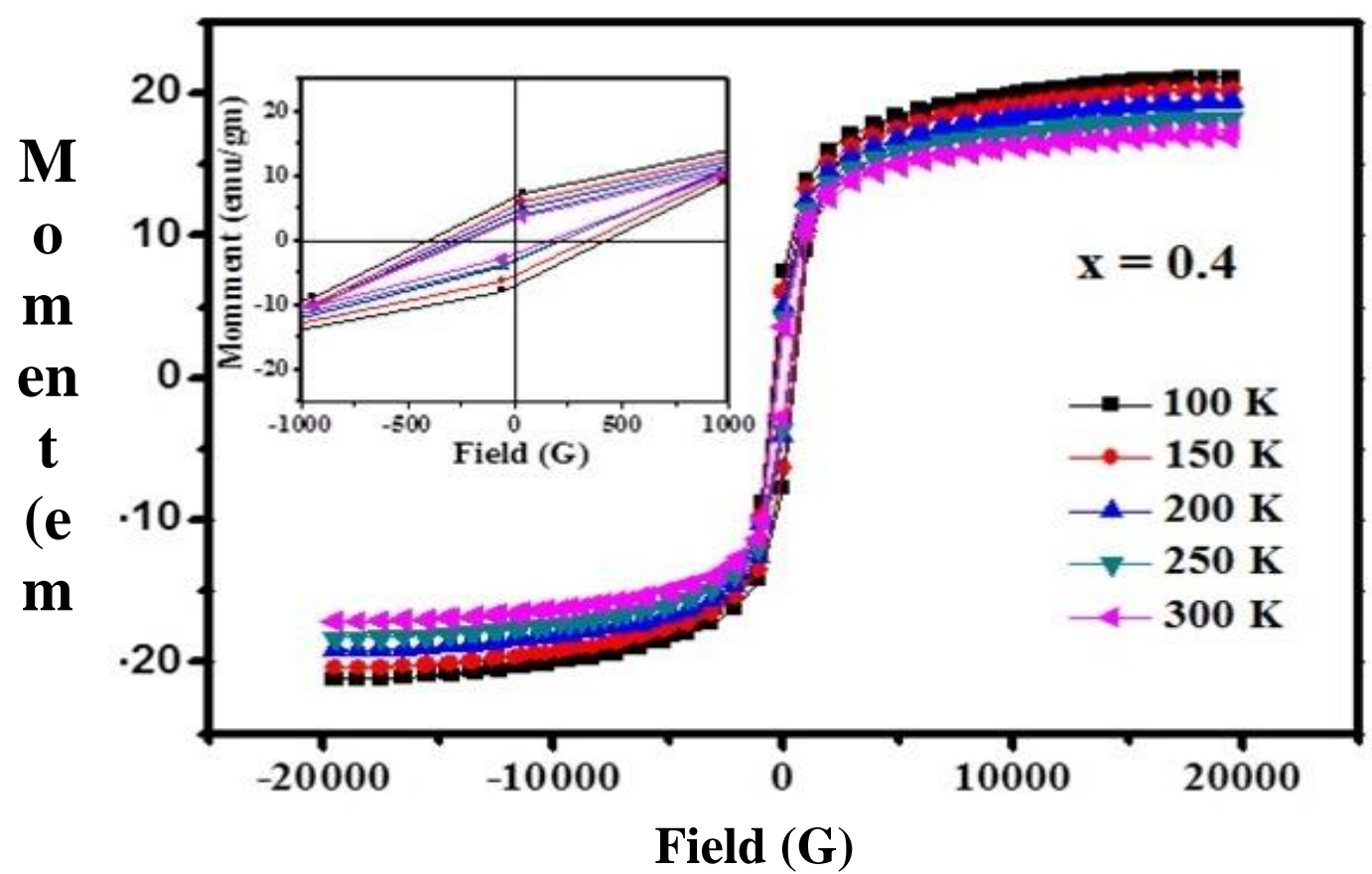

Figure 5: Magnetic hysteresis loops of $x=0.4$ at different temperatures Inset: The middle region of the (VSM) of all temperatures 


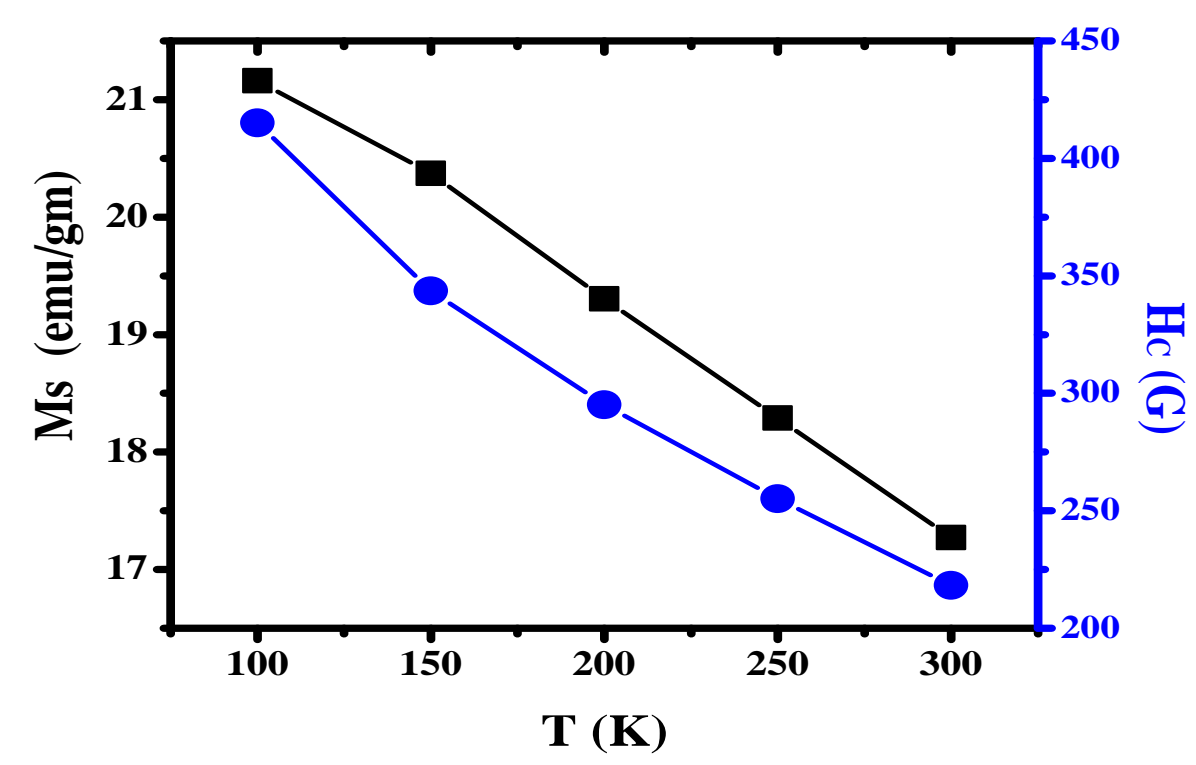

Figure (6): Variation of saturation magnetization $\left(M_{S}\right)$ and coercivity $\left(H_{C}\right)$ for $x=0.4$ at different temperatures

\section{References}

1-Song Yu-Quan, Zhou Wei-Ping, Fang Yong, Yang Yan-Ting, Wang Liao-Yu, Wang Dun- Hui, Du You-Wei, Multiferroic properties in terbium orthoferrite, Chin. Phys. B 23 (7) (2014) 77505.

2-A.A. Azab , N. Helmy, Sukrat Albaaj, Structural and magnetic properties of $\mathrm{La}_{1-\mathrm{x}} \mathrm{Ce}_{\mathrm{x}} \mathrm{Fe}_{1-\mathrm{x}} \mathrm{Cr}_{\mathrm{x}} \mathrm{O}_{3}$ orthoferrite prepared by co-precipitation method, Materials Research Bulletin 66 (2015) 249-253.

3- M.A. Farrukh, Advanced Aspects of Spectroscopy, InTech, 2012, pp. 373.

4-A. Azab and E.H. El-Khawas, Synthesis and Magnetic anomalies of Copper Manganese ferrite Mn1-xCuxFe2O4 (0.0 $\leq \mathrm{x} \leq 0.7)$ Journal of Applied Sciences Research, 9(3)(2013) 1683-1689.

5- M.A.Ahmed, , H.H. Afify, I.K. El-Zawawi and A.A. Azab, Novel structural and magnetic properties of. $\mathrm{Mg}$ doped copper nanoferrites prepared by conventional and wet methods, Journal of Magnetism and Magnetic Materials, 324(2012) 2199-2204.

6-M.B. Bellakki, V. Manivannan, Solution combustion synthesis of - ( $\mathrm{La}, \mathrm{K}) \mathrm{FeO} 3$ orthoferrite ceramics structural and magnetic property studies, Bull. Mater. Sci. 33 (5) (2010) 611.

7-A. Beiranvand, S.M. Hamidi, Z. Abooalizadeh, et al., Life Sci. J.10 (2s) (2013) 181.

8-H. Taguchi, Y. Masunaga, K. Hirota, O. Yamaguchi, Synthesis of perovskite-type $\left(\mathrm{La}_{1-\mathrm{x}} \mathrm{Ca}_{\mathrm{x}}\right) \mathrm{FeO}_{3}(0 \leq \mathrm{x} \leq 0.2) \quad$ at low temperature,Mater. Res. Bull. 40 (2005)773-780.

9-J. P. Zhou, L. Lv, Q. Liu, Y. X. Zhang and P. Liu, Hydrothermal synthesis and properties of
NiFe2O4@BaTiO3composites with well-matched interface, Sci. Technol. Adv. Mater. 13 (2012) 045001.

10- Q. Zhan, R. Yu, S. P. Crane, H. Zheng, C. Kisielowski and R. Ramesh, Structure and interface chemistry of perovskite-spinel nanocomposite thin films. Appl. Phys.Lett., 89, (2006) 172902.

11-A. P. Sutton and R. W. Ballu, Interfaces in Crystalline Materials, Clarendon, Oxford, 1995, p. 240.

12-R. Ramesh, Complex functional oxide heterostructures. Curr. Sci. 105(8), (2013) 11071114.

13-S. Pillai, D. Bhuwal, A. Banerjee and V. Shelke, Bulk interface engineering for enhanced magnetization in multiferroic $\mathrm{BiFeO} 3$ compounds, Appl. Phys. Lett., 102 (2013),

14- J. Hoffmann, S Schnittger, J Norpoth, S Raabe, T Kramer, Ch Jooss, Nanocomposite stability in Fe-, Co-, and Mn-based perovskite/spinel systems, J. Mater. Res., 27(11)(2012),1462-1470.

15-K. Ueda, H. Tabata and T. Kawai, Atomic arrangement and magnetic properties of $\mathrm{LaFeO}_{3}-\mathrm{LaMnO}_{3}$ artificial superlattices, Phys. Rev. B: Condens. Matter Mater. Phys., 60(1999),

Arab J. Nucl. Sci. \& Applic. Vol. 52, No.3 (2019) 
16- Y. B. Chen, J. Zhou, S.-T. Zhang, F.-X. Wu, S.-H. Yao, Z.-B. Gu, D. $\mathrm{Wu}$ and Y.-F. Chen, Significant ferrimagnetisms observed in superlattice composed of antiferromagnetic $\mathrm{LaFeO}_{3}$ and $\mathrm{YMnO}_{3}$, Appl. Phys. Lett.,102(2013), 042403.

17-R. Koferstein and S. G. Ebbinghaus, Synthesis and characterization of nano-LaFeO3 powders by a softchemistry method and corresponding ceramics, Solid State Ionics, 231(2013), 43-48

18-J. Wang, J. B. Neaton, H. Zheng et al., "Epitaxial $\mathrm{BiFeO} 3$ multiferroic thin film heterostructures," Science, 299， 5613(2003),17191722.

19-H. J. Feng, M. Wang, F. Liu, B. Duan, J. Tiam, and X. Guo, "Enhanced optical properties and the origin of carrier transport in $\mathrm{BiFeO}_{3} / \mathrm{TiO}_{2}$ heterostructures with $109^{\circ}$ domain walls," Journal of Alloys and Compounds, 628(2015), 311-316.

20-C. Ederer and N.A. Spaldin, Weak ferromagnetism and magnetoelectric coupling in bismuth ferrite, Phys Rev B 71(2005) 060401-060405.

21- I. Dzyaloshinsky, A thermodynamic theory of "weak" ferromagnetism of antiferromagnetics, J Phys Chem Solids 4(1958)241-255.

22-Moriya T, Anisotropic Superexchange Interaction and Weak Ferromagnetism, Phys Rev 120(1960) 9198.

23-I.Sosnowska, T. Peterlin-Neumaier and Steichele E, Spiral magnetic ordering in bismuth ferrite, J Phys C 15(1982) 4835 4846.

24-T.Y. Kim, N. H. Hong, T. Sugawara, A. T. Raghavender, and M. Kurisu, "Room temperature ferromagnetism with large magnetic moment at low field in rare-earth-doped $\mathrm{BiFeO} 3$ thin films," Journal of Physics: Condensed Matter, 25, (20) (2013), Article ID 206003.

25-A. Scholl, J. Stohr, J. Luning, J.W. Seo, J. Fompeyrine, H. Siegwart, et al., Observation of Antiferromagnetic Domains in Epitaxial Thin Films, Science, 287 (2000) 1014-1016.

26- T.M. Rearick, G.L. Catchen, J.M. Adams, Combined magnetic-dipole and electric-quadrupole hyperfine interactions in rare-earth orthoferrite ceramics.Physyical Review B 48 (1993) 224-238.

27-M.H. Abdellatif, G.M. El-Komy, A.A Azab "Magnetic Characterization of Rare Earth Doped Spinel Ferrite" .Journal of Magnetism and Magnetic Materials, 442 (2017) 445-452.

28- M.H Abdellatif, G.M. El-Komy, A.A Azab, A.M Moustafa "Oscillator strength and dispersive energy of dipoles in ferrite thin film" Materials Research Express, 4, (7)(2017)

29-M.H. Abdellatif, A.A. Azab, and A.M. Moustafa "Dielectric spectroscopy of localized electrical charges in ferrite thin film "Journal of electronic materials, 47(2018)378-384.
30-M.H. Abdellatif, A.A. Azab, M. Salerno "Effect of rare earth doping on the vibrational spectra of spinel Mn-Cr ferrite "Materials Research Bulletin 97 (2018) 260-264

31-M. Sultan, R. Singh, Magnetization and crystal structure of RF-sputtered nanocrystalline $\mathrm{CuFe}_{2} \mathrm{O}_{4}$ thin films, Mater. Lett. 63 (2009) 1764-1766.

32-W. Ponhan, S. Maensiri, Fabrication and magnetic properties of electrospun copper ferrite $(\mathrm{CuFe} 2 \mathrm{O} 4)$ nanofibers, Solid State Science., 11 (2009) 479-484.

33-M. A. Ahmed, A. A. Azab, E. H. El-Khawas, Structural, magnetic and electrical properties of $\mathrm{Bi}$ doped $\mathrm{LaFeO} 3$ nano-crystals, synthesized by autocombustion method, Journal of Materials Science: Materials in Electronics, 26 (2015) 8765-8773.

34- M.A. Ahmed, A. A. Azab, E.H. El-Khawas, E. Abd El Bast, Characterization and transport properties of mixed ferrite system Mn1-xCuxFe2O4; $0.0 \leq \mathrm{x} \leq 0.7$, Synthesis and Reactivity in Inorganic, MetalOrganic, and Nano-Metal Chemistry, 46 (2016) 376384.

35-B.G.Toksha,SagarE.Shirsath, M.L.Mane, K.M.Jadhav "Auto-ignition synthesis of $\mathrm{CoFe}_{2} \mathrm{O}_{4}$ with $\mathrm{Al}^{3+}$ substitution for high frequency applications" Ceramics International 43 (2017) 14347-14353

36- R.D. Shannon,Revised effective ionic radii and systematic studies of interatomic distances in halides and chalcogenides, Acta Crystallogr. A, A32(1976) 751-767.

37-A.A. Azab, Sukrat Albaaj, Effect of grinding time on the structural and magnetic properties of ultrafine $\mathrm{Ni}_{0.7} \mathrm{Zn}_{0.3} \mathrm{Fe}_{2} \mathrm{O}_{4}$, Journal of Ovonic Research, 11(2015) $195-201$.

38-A.A.Azab,S.I. El-Dek,S. Solyman "Unusual features of ferromagnetic/ antiferromagnetic nanocomposites" Journal of Alloys and Compounds. 656 (2016) 987-991

39-Goodenough, J. B. An interpretation of the magnetic properties of the perovskitetype mixed crystals La1xSrxCoO3- $\lambda$. J. Phys. Chem. Solids 1958, 6, 287.

40-Kanamori, J. Superexchange interaction and symmetry properties of electron orbitals. J. Phys. Chem. Solids 10(1959),87-98

41-Gaikwad, V.M.; Acharya, S.A. Novel perovskitespinel composite approach to enhance the magnetization of $\mathrm{LaFeO} 3$. RSC Adv.5(2015)

14366-14373

42-S.Pillai, D.Bhuwal, A. Banerjee and V. Shelke, Appl. Phys.Lett., Bulk interface engineering for enhanced magnetization in multiferroic $\mathrm{BiFeO} 3$ compounds, 102(7) (2013) 072907.

43-Udalov, O. G.; Chtchelkatchev, N. M.; Beloborodov, I. S. Coupling of ferroelectricity and ferromagnetism through Coulomb blockade in composite multiferroics. Phys. Rev. B 89 (2014) 174203. 
44-Belemuk, A. M.; Udalov, O. G.; Chtchelkatchev, N. M.; Beloborodov, I. Competition of magneto-dipole, anisotropy and exchange interactions in composite multiferroics. J. Phys.: Condens. Matter, 28 (2016)

45-Frandsen, C.; Ostenfeld, C. W.; Xu, M.; Jacobsen, C. S.; Keller, L.; Lefmann, K.; Mørup, S. Interparticle interactions in composites of nanoparticles of ferromagnetic $(\gamma$-Fe2O3) and antiferromagnetic $(\mathrm{CoO}, \mathrm{NiO})$ materials. Phys. Rev. B, 70 (2004)

46-M. Mehdipour, H. Shokrollahi, A. Bahadoran, Investigating the exchangecoupling interaction in nanostructure composite particles of $\mathrm{SrFe} 12 \mathrm{O} 19$ and $\mathrm{ZnFe} 2 \mathrm{O} 4$.

J.Electronic Materials, 43(2004) 4282.

47-J.C.G. Tedesco, M.J.M. Pires, A.M.G. Carvalho, V.S.R. de Sousa, L.P. Cardoso, A.A. Coelho, Exchange-bias-like effect in $\operatorname{Pr} 0.75 \mathrm{~Tb} 0.25 \mathrm{Al} 2$ and Pr0.7Tb0.3Al2 samples., J. Magn. Magn. Mater. 339 (2013) 6-10.

48-Amir Aslani, Mohammadreza Ghahremani, Ming Zhang, Lawrence H. Bennett, Edward Della Torre, Enhanced magnetic properties of yttrium-iron nanoparticles,

AIP Advances 7 (2017) 056423.

49- Koji Yoshida, Yuki Fujii, Hajime Shimizu, Reduction of Curie temperature and saturation magnetization in Ba2-xNaxFeMoO6 J. Appl. Phys. 98 ( 2005) 103901.

50-M. Kiwi, Exchange bias theory, J. Magn. Magn. Mater. 234 (2001) 584-595.

51- J. Nogués, I.K. Schuller, Exchange bias, J. Magn. Magn. Mater. 192 (1999) 203-232.

52-S. Sabyasachi, M. Patra, S. Majumdar, S. Giri, Constricted double loop hysteresis and exchange bias attributed to the surface anisotropy in nanocrystalline
La1/3Sr2/3Fe1-xCrxO3', J. Magn. Magn. Mater. 344 (2013) 20-24.

53- R.N. Bhowmik, V. Vasanthi, A. Poddar, Alloying of $\mathrm{Fe}_{3} \mathrm{O}_{4}$ and $\mathrm{Co}_{3} \mathrm{O}_{4}$ to develop $\mathrm{Co}_{3 \mathrm{x}} \mathrm{Fe}_{3(1-\mathrm{x})} \mathrm{O}_{4}$ ferrite with high magnetic squareness, tunable ferromagnetic parameters, and exchange bias J. Alloys Compd. 578 (2013) 585-594.

54- G. Yuqiao, S. Lei, Z. Shiming, Z. Jiyin, W. Cailin, L. Wenjie, W. Shiqiang, Tunable exchange bias effect in Sr-doped double perovskite La2NiMnO6., J. Phys. D: Appl. Phys. 46 (2013) 175302.

55- Y.Y. Wang, C. Song, F. Zeng, F. Pan, "Fielddirection sensitive magnetization reversal in a perpendicularly exchange-coupled system" J. Phys. D: Appl. Phys. 46 (2013) 445001.

56-B.M. Tanygin, "On the free energy of the flexomagnetoelectric interactions" J. Magn. Magn. Mater. 323 (2011) 1899-1902.

57-F. Hong-Jian "Spin transfer in ultrathin BiFeO3 film under external electric field" EPL 101 (2013) 67007.

58-P.K. Manna, S.M. Yusuf, B. Mrinmoyee, P. Tarasankar" he magnetic proximity effect in a ferrimagnetic $\mathrm{Fe} 3 \mathrm{O} 4$ core/ferrimagnetic $\gamma$-Mn2O3 shell nanoparticle system" J. Phys.: Condens. Matter 23 (2011) 506004.

59- B. M. Wang, Y. Liu, P. Ren, B. Xia, K. B. Ruan, J. B. Yi, J. Ding, X. G. Li, L. Wang, Large Exchange Bias after Zero-Field Cooling from an Unmagnetized State, Phys. Rev. Lett. 106 (2011) 077203.

60-Yue Huang, Song Li, Zhanqiang Tian,et al. Strong room temperature spontaneous exchange bias in $\mathrm{BiFeO}_{3}-\mathrm{CoFe}_{2} \mathrm{O}_{4}$ nanocomposites. J. Alloys Compd. 762 (2018) 438-443

61- Sarkar Tanushree, Suja Elizabeth, P.S. Anil Kumar Electron doping induced exchange bias and cluster glass magnetism in multiferroic $\mathrm{Sc}_{0.8} \mathrm{Zr}_{0.2} \mathrm{MnO}_{3}$, J. Magn. Magn. Mater. 466 (2018 ) 225. 